\title{
Study on The Mildewproof Method of Fresh Xinhui Citrus Peels
}

\author{
Chen LI* \\ School of Chemistry and Environmental Engineering \\ Wuyi University \\ Jiangmen 529020, China \\ e-mail:wyuchemlc@126.com \\ Jingyi MA \\ School of Chemistry and Environmental Engineering \\ Wuyi University \\ Jiangmen 529020, China \\ e-mail: 396322207@qq.com
}

\author{
Yingzhu WU \\ School of Textile and Clothing \\ Wuyi University \\ Jiangmen 529020, China \\ e-mail: 372550116@qq.com
}

\author{
Leyu MA \\ School of Chemistry and Environmental Engineering \\ Wuyi University \\ Jiangmen 529020, China \\ e-mail: 492329659@qq.com
}

\author{
Ning QING \\ School of Chemistry and Environmental Engineering \\ Wuyi University \\ Jiangmen 529020, China \\ e-mail:wyuchemqn@126.com
}

\begin{abstract}
To explore the effect to storage method of fresh Xinhui citrus peels by using against storage and ethanol mildewproof method, the main fungus which caused the mildew of fresh peels was also checked. The effect of hance ginger on peel moldy was observed by changing the humidity of storage environment of citrus peels; the effects of storage conditions were also observed using different concentrations of ethanol sprayed on fresh peel, and the effects of ethanol on the flavonoids of fresh citrus peel during storage. Both the hance ginger against storage and ethanol against storage methods can effectively delay the mildew of fresh citrus peels, the latter method can effectively inhibit the mildew. The main fungi produced by microbial culture experiment showed that mildew after is Penicillium and Aspergillus niger.
\end{abstract}

Keywords-Xinhui citrus peels; mildewproof; fungus; flavonoids

\section{INTRODUCTION}

Xinhui Pericarpium Citri Reticulatae, containing of flavonoids [1-2], volatile oil [3-4], polysaccharide, yellow pigment, dietary fiber etc. active components, has anticancer, anti allergy, cough and other pharmacological effects [5-9]. The fresh peels of Xinhui Citrus reticulata $c v$. Chachiensis of Guangdong province is the raw materials of Xinhui tangerine peel, washed, dried, cut, sun-dried and natural storage for three years than the peels will become Pericarpium Citri Reticulatae [10]. However, because of Guangdong's hot and humid climate, during the drying and storage of fresh citrus peels, the improper storage of citrus peels often resulting in moldy, thus affecting the quality of Xinhui Pericarpium Citri Reticulatae [11]. It was reported that some traditional Chinese medicines (TCMs) against storage belongs to the biological control of mildew category, these TCMs have some special components and could emit special smell which can mildewproof and insect repellent. When stored with these against TCMs together, the biological materials which easy to mold so as to achieve anti moth and mildew [12-13]. Chemical preservatives [14] and tangerine peel essential oil [15] have been used to the preservation of citrus. Ethanol is often used as a reagent for sterilization and disinfection, and it is environment friendly, even if there is residual will not cause harm to human body. It can make the protein coagulation degeneration by using ethanol incorporated into the cell, so as to achieve the effect of sterilization and bacteria. Both of the mildewproof effects to Xinhui citrus peels, against storage method (hance ginger) and ethanol against method, were respectively illuminated in this paper.

\section{EXPERIMENTAL}

\section{A. Instruments, Materials and Reagents}

Finnigan Surveyor High performance liquid chromatograph (HPLC) equipped with an automatic injector, four element pump, photodiode array (PDA) detector, workstation (USA, Thermo Scientific); 759S UV visible spectrophotometer (Shanghai Lengguang Technology Co Ltd) ; KQ-30VDV ultrasonic cleaning (Kunshanultrasonic instruments Co. Ltd.); electronic balance (Changshu Shuangjie testing instrument factory); XSP-20A type microscope (Changshu Shuangjie testing instrument factory); loop (Ohaus instrument company).

Xinhui Citrus (the origin of the Sanjiang town of Guangdong Xinhui District); transparent sealed storage tank (with pressure-sensitive pads, $10 \mathrm{~cm}$ high, $9.5 \mathrm{~cm}$ in 
diameter); hygrothermograph (Guangbo stationery); hance ginger (purchased from pharmacies).

Ethanol (AR, 500mL, Shantou Xilong Chemical Factory); methanol (HPLC grade, 4 L, Merck Co); acetic acid (HPLC grade, $1 \mathrm{~L}$, Fluka); modified Martin medium (Hangzhou Baisi Biotechnology Co. Ltd.); lactic acid phenol cotton blue staining solution (Xiamen Haibiao Science and Technology Co., Ltd.); hesperidin and nobiletin (Beijing Hengyuan Qitian Chemical Technology Research Institute).

\section{B. Hance Ginger against Storage Test}

The hance ginger was chosen as the against herb for its cheapness and unique smell, by changing the water spray quantity on the surface of frseh Xinhui citrus peels, the mildewproof role under different humidity of fresh fruit peel was observed. The operation procedure was as follows: a fresh citrus were well cleaned with water, then dried and cut open to three pieces with knife. Six pieces of similar size citrus peels were chosen using four point methods, including one peel naturally placed as blank, the other five peels were separately placed in five transparent sealed vials. At position $2 \mathrm{~cm}$ from the fresh peel samples, $0 \mathrm{~mL}, 0.6 \mathrm{~mL}, 1.2 \mathrm{~mL}$, $1.8 \mathrm{~mL}$ and $4.2 \mathrm{~mL}$ pure water was respectively and evenly sprayed on the surface of fresh citrus peels with a hand-held compression sprayer, and $10 \mathrm{~g}$ hance ginger placed together in the same sealed vial with the sprayed peel. $30 \mathrm{~mL}$ water was also placed in the vial in order to speed up the process of mildew of fresh peel. A hygrometer was placed in each vial for daily observation records in the process of storage temperature and humidity and mildew.

\section{Ethanol against Test}

The effects of ethanol solution of different concentration on the mildewproof abilities of fresh citrus peels were studied. The specific operation was as follows: fresh citrus was cleaned, dried and cut open into three pieces. Ethanol solution with $55 \%, 75 \%, 95 \%$ ethanol were prepared by adding pure water into ethanol, then respectively sprayed on three inner and outer fresh citrus peels with similar size. Keeping the whole peel was wet, and then in three seal pocket bags each, and $30 \mathrm{~mL}$ ethanol solution of corresponding concentration was put into the same vial. Observation and record the situation of mildew during the storage process. Storage ambient temperature was maintained at $20-22{ }^{\circ} \mathrm{C}$, humidity $50-60 \%$.

\section{Preparation of Flavonoids Extract}

Weighing $1 \mathrm{~g}$ (accurate to $0.01 \mathrm{~g}$ ) Xinhui citrus peel sample dried or stored for a certain time, placed in a $250 \mathrm{~mL}$ conical flask, $60 \mathrm{~mL}$ of $60 \%$ methanol solution $(\mathrm{v} / \mathrm{v})$ was added in the flask, after $30 \mathrm{~min}$ immersion, then extracted 30 min in an ultrasonic cleaner at extraction power $45 \%$, temperature $65^{\circ} \mathrm{C}$, ultrasonic frequency $45 \mathrm{kHz}$, filtrated in succession, the filtrate was concentrated $\left(50-60^{\circ} \mathrm{C}\right)$ until the volume of $50 \mathrm{~mL}$, finally the flavonoids extract from citrus peels was obtained.

\section{E. Analysis of Flavonoids}

1) Hesperidin and nobiletin -- HPLC method: The flavonoids extract was filtered using 0.22 micron membrane and then analysed with HPLC. Chromatographic conditions was as follows: Hypersil Gold column, size $150 \times 2.1 \mathrm{~mm}, 3.5$ micron particle (American Thermo company); mobile phase: $2 \%(\mathrm{v} / \mathrm{v})$ acetic acid solution of A phase, HPLC grade methanol of $\mathrm{B}$ phase, gradient elution procedures: $30 \% \mathrm{~B}$ evenly increased to $70 \% \mathrm{~B}(1-25 \mathrm{~min})$, then $70 \% \mathrm{~B}$ evenly reduced to a $30 \% \mathrm{~B}(25-30 \mathrm{~min})$, a total analytical time of $30 \mathrm{~min}$; flow rate: $0.3 \mathrm{~mL} / \mathrm{min}$; column temperature: $25^{\circ} \mathrm{C}$; detection wavelength: $283 \mathrm{~nm}$; sample size: 10 microlitre.

2) Total flavonoids -- UV method: The flavonoids extract was diluted 100 times with methanol, and the total flavonoids in the UV test solution were obtained. With pure methanol as the reference solution, and hesperidin as the control standard, the detection wavelength was set at $283 \mathrm{~nm}$. The standard curve of hesperidin was prior obtained, then the total flavonoids content of the dry Xinhui Citrus peels would be calculated under the same test conditions.

\section{F. Mildew Fungi Culture and Microscopic Examination}

Experimental methods: $2.85 \mathrm{~g}$ Martin medium and $1.5 \mathrm{~g}$ agar were weighed and poured into the beaker, and then $100 \mathrm{~mL}$ pure water was added. The beaker was then placed on the electric heating board for heating, and keeps stirring until completely dissolved. After the dissolution, the medium was poured into the conical flask and placed in the sterilizer of $115^{\circ} \mathrm{C}$ for $30 \mathrm{~min}$. After sterilization, the medium was slightly cooled to about $50{ }^{\circ} \mathrm{C}$, and then poured into a culture dish (about 10 to $15 \mathrm{~mL} /$ dish) above the alcohol lamp flame, to be thin and smooth. Using the inoculation loop, three different inoculation position of the colony from the peel of five level mildew was then respectively chosen. And then the culture medium was placed in the $25^{\circ} \mathrm{C}$ biochemical incubator for 3 days. After the training completed, the fungi were checked by the microscopy. On the slide with a drop of lactic acid phenol cotton blue staining solution, the index finger and thumb stuck were stuck on both ends of a transparent adhesive tape. The tape shape was $U$ type, glue face down, gently touching the surface of the mildew, which was then dipped into the lactic acid phenol cotton blue staining solution slides, and the tape ends were fixed on both ends of the slide, finally checked under low magnification, then high magnification by biological microscope.

\section{RESULTS AND DISCUSSION}

\section{A. Result of Hance Ginger against Storage}

According to the sensory observation of the moldy level of the citrus peels, the mildew peels could be divided into five levels, that is level 1 to level 5 corresponding from mild to severe, as shown in Fig. 1. Fig. 2 shows the mildew levels of fresh citrus peels with hance ginger in the against storage test. The blank group was peels without hance ginger, group 1 was no water sprayed, group 2 was $0.6 \mathrm{~mL}$ water sprayed, group 3 was $1.2 \mathrm{~mL}$ water sprayed, group 4 was $1.8 \mathrm{~mL}$ water sprayed, and group 5 was $4.2 \mathrm{~mL}$ water sprayed. 


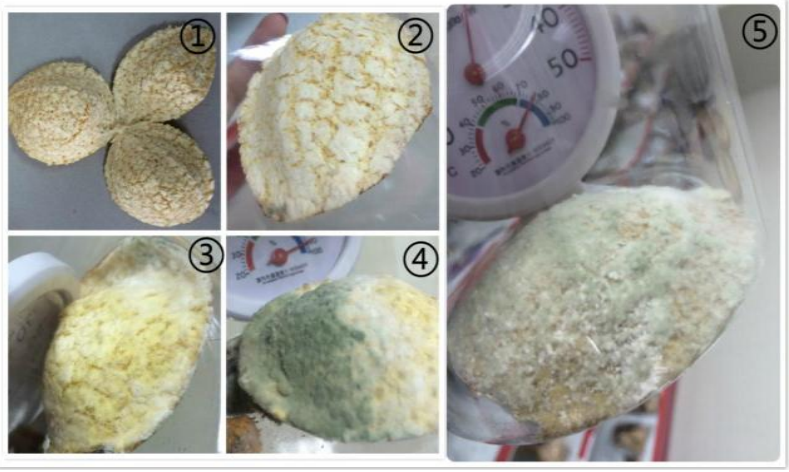

Figure 1. The graph of mildew level of the fresh citrus peel (1)no mildew; (2)began to mildew; (3)slight mildew; (4)medium mildew; (5)serious mildew

It could be found that whether or not to join the against herb hance ginger, fresh citrus peel would begin to mildew when placed at room temperature for 8 days, but moldy degree and speed of the blank group of fresh fruit peel without hance ginger was more serious. The moldy of fresh peel of blank group had reached level 5 after 12 days. Store together with fresh peel and hance ginger, the mildew occurrence time was slightly later, more water volume sprayed onto the peel, more serious mildew occurred. For example, the group 1 to group 3 was less water sprayed, eventually reach level 3 . However, the group 4 and group 5 which had more water sprayed reached level 4 of mildew. In addition, the hance ginger water sprayed placed alone in sealed veal, only after 2 days, the mildew could be found. Therefore, when stored together with hance ginger, whether or not water sprayed, the mildew degree of fresh citrus peel could be slowed, hance ginger and citrus peel can against each other in the inhibition of fungal growth can help to extend the shelf life of fresh citrus peel. Fig. 3 were the comparison pictures of fresh citrus peel with hance ginger together stored for 1 day (upper) and 14 days (below) in the herb against test.
TABLE I. EFFECT OF DIFFERENT ETHANOL SOLUTION SPRAYED ON FRESH Citrus PeEL

\begin{tabular}{ccccc}
\hline $\begin{array}{c}\text { Sample } \\
\text { No. }\end{array}$ & Peel processing & $\begin{array}{c}\text { Total } \\
\text { flavonoids } \\
\text { content } \\
\text { \% }\end{array}$ & $\begin{array}{c}\text { Hesperidin } \\
\mathbf{~} \mathbf{m} \cdot \mathbf{L}^{-\mathbf{1}}\end{array}$ & $\begin{array}{c}\mathbf{N o b i l e t i n} \\
\mathbf{m g} \cdot \mathbf{L}^{-1}\end{array}$ \\
\hline 1 & $\begin{array}{c}\text { Untreated control } \\
\text { group }\end{array}$ & 1.69 & 370.37 & 21.96 \\
2 & $55 \%$ ethanol spray & 2.55 & 434.83 & 30.16 \\
3 & $75 \%$ ethanol spray & 1.52 & 234.14 & 37.10 \\
4 & $95 \%$ ethanol spray & 0.60 & 184.30 & 29.74 \\
5 & $\begin{array}{c}\text { Ethanol } \\
\text { precipitation } \\
\text { liquid }\end{array}$ & 1.78 & 262.43 & 148.65 \\
\hline
\end{tabular}

\section{B. Result of Ethanol against Test}

It was found that when sprayed with the ethanol solution, the fresh citrus peel could be long-term stored in the sealed vial (about 150 days). At the same time, it was found that the ethanol solution level in the sealed vial decreased during the storage period, along with the scented and orange liquid appeared in the transparent sealing pocket within the citrus peel. This may be due to high concentration of ethanol volatile, gaseous ethanol would be permeated into the fresh peel, thus made the alcohol soluble components precipitation. And higher content of ethanol solution sprayed, more ethanol precipitation liquid appeared. The contents of hesperidin and nobiletin were determined using HPLC method above described, and the total flavonoids was determined using UV-vis method above mentioned. The determination results of fresh citrus peel sprayed by different concentration of ethanol solution $(55 \%, 75 \%$, and $95 \%$ ethanol, v/v), along with the untreated fresh peel were all shown in Table I.

Considering the loss of flavonoids in the ethanol precipitation liquid, the extracted liquid in the sealing pocket was also checked for total flavonoid (UV) and two above flavonoid compounds (HPLC), the results were also listed in Table I.

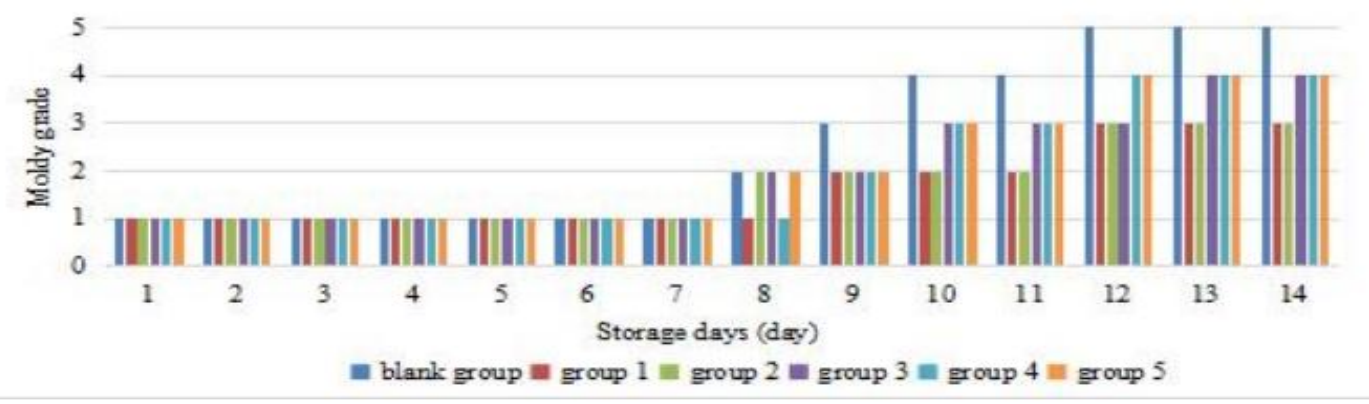

Figure 2. Result of against storage test using hance ginger with fresh peels 


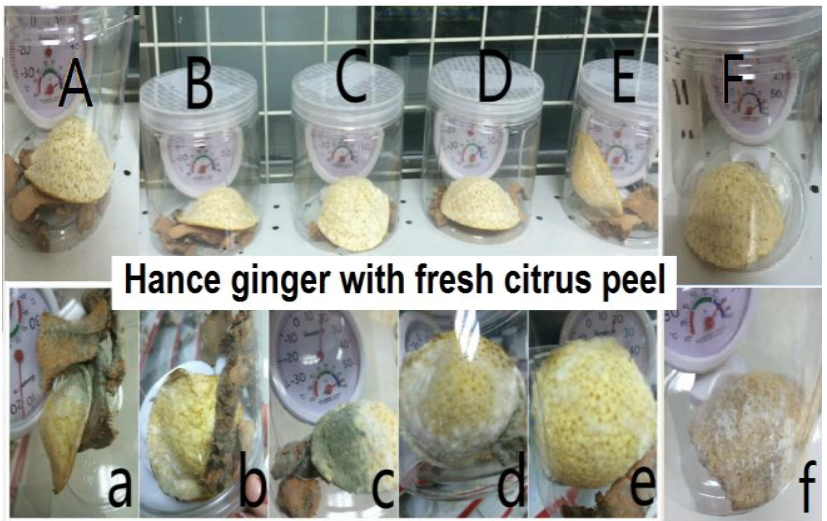

Figure 3. Pictures comparison of the against storage test A to $\mathrm{E}$, a to e: corresponding to group 1 to group 5 in Fig.2, F and f: blank group without hance ginger, Upper pictures: stored only for 1 day, below pictures: stored for 14 days.

It was shown in Table I that the content of flavonoids in citrus peels significantly changed when treated by the ethanol solution. Compared to the untreated peel, 55\% ethanol treated peel could be extracted of much more contents of hesperidin, nobiletin and total flavonoids, indicating the proper concentration of ethanol solution after spraying was effective for the precipitation and extraction of flavonoids. However, when ethanol content was further increased to $75 \%$ or $95 \%$, the contents of total flavonoids and hesperidin in citrus peel were both less than the untreated control group, with the increase of the content of ethanol content decreased more. It could be due to the polarity of hesperidin is larger, but the polarity of high concentration of ethanol is lower. According to the theory that similarities can be solvable easily in each other, so the extract was rather lower than untreated peel. On the other hand, it was due to high concentration of ethanol had more volatile ability, so more of the precipitation liquid found on the peel surface, resulting in the loss of flavonoids in peels, which can be confirmed from the results in Table I.

In summary, ethanol against method can effectively prevent the mildew of fresh peel, but the concentration of ethanol and the reasonable dosage must be controlled, otherwise it will make the quality decline of Pericarpium Citri Reticulatae. Therefore, 55\% ethanol solution can be chosen as a inhibitor sprayed on the fresh citrus peels, which can effectively delay the mildew of the fresh peel, not loss of flavonoids, but improve the extraction rate of flavonoids from citrus peels.

\section{Fungal Culture and Microscopic Examination}

The raw material (Fig. 4a) was chosen from the severe mildew of levels 5; a cultured microorganism colony (Fig. $4 \mathrm{~b}$ ) was prepared by cultivation method described in section $\mathrm{F}$ of Experimental part. The fungal morphology of Fig. 4c and Fig.4d were observed. From the fungal morphology of cultured mildew, the Penicillium bacteria was identified for the features of felted or loose floc, mostly gray and green, the top was not inflated, etc, roughly the same as shown in Fig. 4c. The vegetative hyphae of Aspergillus Niger has
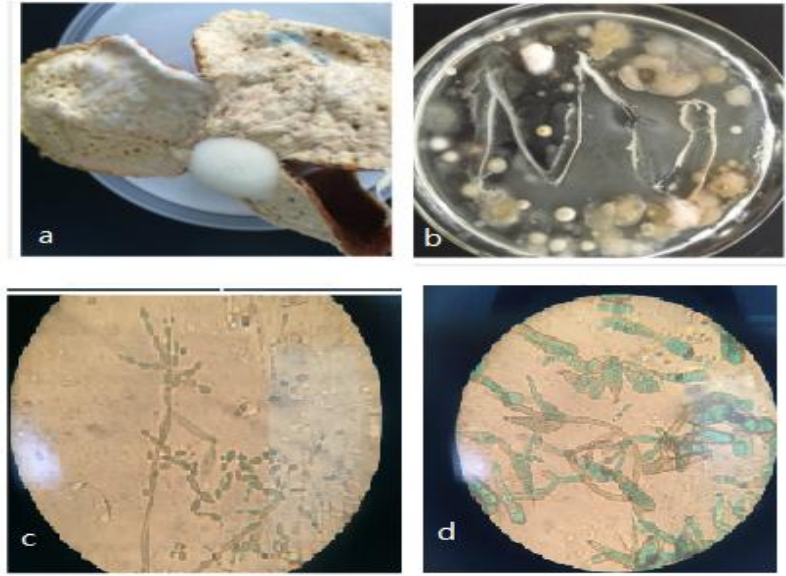

Figure 4. Pictures (a and b) and microscopy check (c and d) of the fungal culture test

separated chypha forming part of a growing and rough conidiophores, tip swelling forming a spherical vesicle, the surface radiation of one or two layers of small stems, small spherical stalk formation on the conidia, closed to the Fig. 4d graphics. So it could be judged as mildew fungi Penicillium (Fig. 4c) and Aspergillus niger (Fig. 4d), which in common with the results in [16-17]. WANG Fu [17] also pointed out that the main fungi which increased the content of flavonoid were Aspergillus niger in the citrus peel.

\section{SUMMARY}

The following conclusion could be obtained:

(1) The special volatile substances of hance ginger were used to effectively prolong the shelf life of fresh citrus peel in suitable humidity conditions, and the degree of mildew peels reduced.

(2) The 55\%-95\% ethanol solution could prevent mildew of fresh citrus peel (for 150 days), but high content of ethanol (higher than $75 \%$ ) sprayed on the peel would cause the loss of flavonoids in the peel. So the $55 \%$ ethanol solution was recommend to be sprayed on the citrus peels, not only few loss of flavonoids in the peel, but also much more increase of the extraction rate of flavonoids.

(3) The microbial culture and microscopy results showed that the main fungi from fresh Xinhui citrus peels were Penicillium and Aspergillus niger.

\section{ACKNOWLEDGMENT}

The authors gratefully acknowledge the financial support provided by Science and Technology Department of Guangdong Province Project (2015A020209194), Agricultural science and technology project of Jiangmen City (Jiang Caigong [2014] No. 173), Philosophy and Social Sciences Planning in Jiangmen (JM2015C03).

\section{REFERENCES}

[1] Wang DD, Wang $\mathrm{J}$, Huang $\mathrm{XH}$, et al. Identification of polymethoxylated flavones from green tangerine peel ( Pericarpium Citri Reticulatae Viride) by chromatographic and spectroscopic 
techniques [J], Journal of Pharmaceutical and Biomedical Analysis, 2007, 44( 1): 63 -69.

[2] E-Hu Liu, Pan Zhao, Li Duan, et al. Simultaneous determination of six bioactive flavonoids in Citri Reticulatae Pericarpium by rapid resolution liquid chromatography coupled with triple quadrupole electrospray tandem mass spectrometry [J]. Food Chemistry, 2013, 141: 3977-3983.

[3] Yamin Wang, Lunzhao Yi, Yizeng Liang, et al. Comparative analysis of essential oil components in Pericarpium Citri Reticulatae Viride and Pericarpium Citri Reticulatae by GC-MS combined with chemometric resolution method[J]. Journal of Pharmaceutical and Biomedical Analysis, 2008, 46 :66-74.

[4] OU Xiao-qun, WANG Jin, LI Peng, et al. Comparison among volatile oil compositions from tangerine peels and their kindreds [J]. Chinese Traditional Patent Medicine, 2015, 37(2): 364-370.

[5] Li Duan, Long Guo, Li-Li Dou, et al. Discrimination of Citrus reticulata Blanco and Citrus reticulata 'Chachi' by gas chromatograph-mass spectrometry based metabolomics approach [J]. Food Chemistry, 2016, 212: 123-127.

[6] YUAN Han-qi, HUANG Qing-hua, ZHANG Xiao-ying, et al. Intestinal absorption properties in rats of Nobiletin and Tangeretin in ethanol extract of Citrus reticulata 'Chachi' peel by single pass perfusion model [J]. Food Science, 2014, 35(15): 263-267.

[7] Lun-zhao Yi, Da-lin Yuan, Yi-zeng Liang, et al. Fingerprinting alterations of secondary metabolites of tangerine peels during growth by HPLC-DAD and chemometric methods [J]. Analytica Chimica Acta, 2009, 649: 43-51.

[8] Lunzhao Yi, Naiping Dong, Shao Liu, et al. Chemical features of Pericarpium Citri Reticulatae and Pericarpium Citri Reticulatae Viride revealed by GC-MS metabolomics analysis [J]. Food Chemistry, 2015, 186: 192-199.
[9] Su-Chen Ho, Chun-Ting Kuo. Hesperidin, nobiletin, and tangeretin are collectively responsible for the anti-neuroinflammatory capacity of tangerine peel (Citri reticulatae pericarpium) [J]. Food and Chemical Toxicology, 2014, 71: 176-182.

[10] Product of geographical indication - Xinhui mandarin orange peel[S] DB44/T 604-2009.

[11] Ortuno A, Diaz L, Alvarez N, et al. Comparative study of flavonoid and scoparone accumulation in different Citrus species and their susceptibility to Penicillium digitatum [J], Food Chemistry, 2011, 125(1) : 232-239.

[12] Sun Shu-ying. The experience of Chinese medicine mothproof [J], Heilongjiang Journal of Traditional Chinese Medicine, 2001, 5: 60.

[13] Sun Shu-jie, Han Xiao-jie, Li Wen-xiang, et al. Effect of Licorice or Hance Ginger and Its Complex Extract on the Fresh-Keeping of Spinach [J], Journal of Food Science and Biotechnology, 2012, 31(5):537-542.

[14] ZHANG Yan, HOU SHI-kui, ZHANG Yi-gang,et al. Effects of different chemical preservation agents on W.Murcott during storage [J], Storage and Process, 2010, 10(6): 15-17.

[15] Qi He, Kaijun Xiao. The effects of tangerine peel (Citri reticulatae pericarpium) essential oils as glazing layer on freshness preservation of bream (Megalobrama amblycephala) during superchilling storage [J]. Food Control, 2016, 69: 339-345.

[16] Zhang Liang, Zhou Jie, Zhu Wei, et al. Comparative analysis on the chemical components of pericarpium Citri Reticulatae, citrus peels and fermented citrus peels with HPLC-DAD-ESI-MSn and GC-MS [J]. Food and Fermentation Industries, 2013, 39(4):192-199.

[17] WANG Fu, ZHANG Xin, LU Jun-yu, et al. Study of reason "ofthe older, the better" of Pericarpium Citri Reticulatae (PCR) which contributed increase of flavonoids [J], China Journal of Chinese Materia Medica, 2015, 40(24):4890-4896. 